ratio of bubble to oil in the two-phase droplet $\left(\beta_{i}\right)$ and the volume equivalent sphere diameter $(d)$ are expressed by Eq.(3) and Eq.(4).

$$
\begin{aligned}
& \beta=\beta_{i} \frac{P}{P-\rho_{w} \cdot y\left(g / g_{c}\right)} \\
& d=d_{i}\left(\frac{1+\beta}{1+\beta_{i}}\right)^{1 / 3}
\end{aligned}
$$

Figures 3, 4 and 5 are shown to determine the constants $A, k_{1}, k_{2}$ and $k_{3}$.

As the result, the final correlation for H.T.U. based on dispersed phase $\left(H_{\dot{d}}\right)$ is shown in Fig. 5, and is expressed by the equation

$$
\left(\frac{H_{d}}{d}\right) \cdot N_{\mathrm{C} a}=3.80 \times 10^{-5} \cdot N_{P e}^{1 / 2}
$$

The equation agrees satisfactorily with previous ex-

\begin{tabular}{|c|c|c|}
\hline$A$ & $=$ & constant in Eq.(2) \\
\hline$C$ & $=$ & solute concentration of oil \\
\hline$C_{i}$ & $=$ & solute concentration of oil initially \\
\hline $\mathscr{D}$ & $=$ & diffusion coefficient \\
\hline$d$ & $=$ & volume equivalent sphere diameter of droplet \\
\hline$d_{b, i}$ & $=$ & $\begin{array}{l}\text { volume equivalent sphere diameter of } \\
\text { bubble in two-phase droplet at } \\
\text { tip of nozzle }\end{array}$ \\
\hline$d_{i}$ & $=$ & $\begin{array}{l}\text { volume equivalent sphere diameter of } \\
\text { droplet at tip of nozzle }\end{array}$ \\
\hline$d_{N I}$ & $=$ & inside diameter of nozzle \\
\hline$H_{d}$ & $=$ & $\begin{array}{l}\text { height per transfer unit based on dispersed } \\
\text { phase for mass transfer }\end{array}$ \\
\hline$k_{1}$ & $=$ & exponent in Eq.(2) \\
\hline
\end{tabular}
perimental data ${ }^{1,3)}$.

\section{Nomenclature}

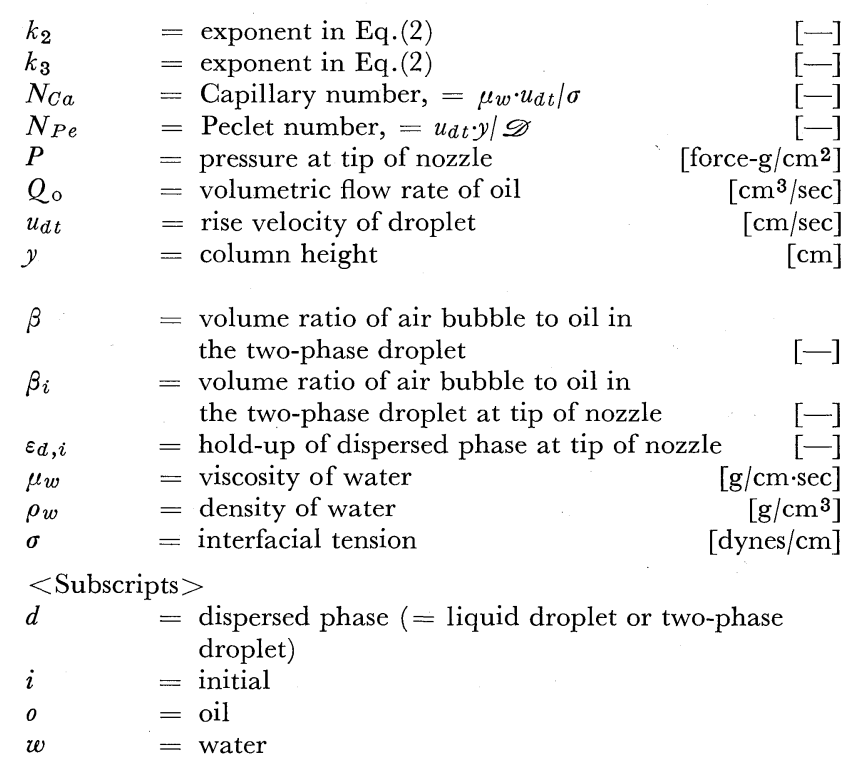

\section{Literature Cited}

1) Fujinawa, K. and Y. Nakaike: Kagaku Kōgaku (Chem. Eng., Japan), 22, 540 (1958)

2) Hayakawa, T. and M. Shigeta: J. Chem. Eng. Japan, 6, 273 (1973) also see Hayakawa, T. and M. Shigeta: Kagaku Kōgaku, 38, 66 (1974)

3) Hayashi, Y. and H. Inazumi: Preprint of the 6th Autumn Meeting of The Soc. of Chem. Engrs., Japan, B-110, Tokyo (1972)

4) Higbie, R.: Trans. AIChE., 31, 365 (1935)

5) Ruby, C. L. and J. C. Elgin: Chem. Eng. Prog. Symposium Ser., 51 (16), 17 (1955)

\title{
CHROMATOGRAPHIC MOMENTS FOR PACKED BED REACTORS AND BOUNDARY CONDITION AT BED EXIT*
}

\author{
Noriaki WAKAO, Kenji TANAKA and D. S. SCOTT** \\ Department of Chemical Engineering, Yokohama National University, Yokohama, Japan
}

In estimating kinetic and transport parameters in the packed bed reactor the chromatographic technique, which requires exact solution for tracer output in Laplace domain, is becoming popular.

When the tracer is injected deep in a bed free from bed-inlet effect, there are the following two cases regarding the locati $n$ of measurement of output signal:

Case 1: Output signal is measured at bed exit.

Case 2: Output signal is measured in bed and the bed extends sufficiently far downstream beyond the measurement point.

Kubin $^{1)}$ and Kucera ${ }^{2)}$ have obtained Eq.(11). The purpose of this note is to obtain $t$ e moments for Case

\footnotetext{
* Received on July 12, 1973

** Dept. of Chem. Eng., University of Waterloo, Waterloo, Ontario, Canada 厂233 横浜市南区大岡2-31-1

横浜国立大学工学部化学工学科 若尾法昭
}

1, considering Danckwerts' exit-boundary condition. The particles are assumed to be porous and f uniform size.

\section{(A) Homogeneous Reaction Model}

This is the model for catalyst particles. It is assumed that the reaction in the particles is of isothermal first order with regard to the imposed reactant.

The system is expressed as

$$
\begin{gathered}
\frac{\partial C}{\partial \tau}=\beta \frac{\partial^{2} C}{\partial x^{2}}-\frac{\partial C}{\partial x}-\frac{3 \eta}{\kappa}\left(\frac{\partial c_{i}}{\partial \rho}\right)_{\rho=1} \\
\kappa \frac{\partial c_{i}}{\partial \tau}=\frac{\partial^{2} c_{i}}{\partial \rho^{2}}+\frac{2}{\rho} \frac{\partial c_{i}}{\partial \rho}-\phi^{2} c_{i} \\
C(x, 0)=0 \\
c_{i}(\rho, x, 0)=0
\end{gathered}
$$


Table 1 Comparison of moments for homogeneous reaction model

Case 1

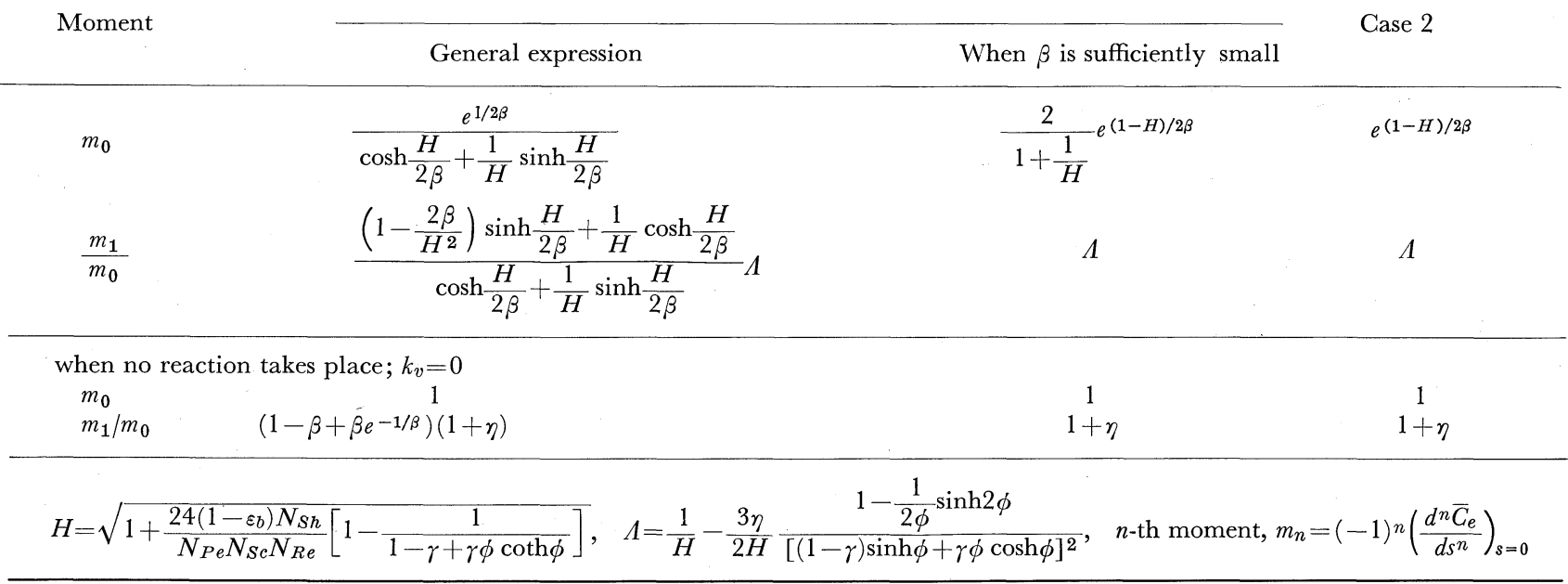

Table 2 Comparison of moments for shell reaction model

\begin{tabular}{|c|c|c|c|}
\hline \multirow{2}{*}{ Moment } & & & \multirow{2}{*}{ Case 2} \\
\hline & General expression & When $\beta$ is sufficiently small & \\
\hline \multirow[b]{2}{*}{$m_{0}$} & $e^{1 / 2 \beta}$ & $2-e^{(1-T) / 2 \beta}$ & $e^{(1-T) / 2 \beta}$ \\
\hline & $\cosh \frac{T}{2 \beta}+\frac{1}{T} \sinh \frac{T}{2 \beta}$ & $1+\frac{1}{T}$ & \\
\hline$\frac{m_{1}}{m_{0}}$ & $\frac{\left(1-\frac{2 \beta}{T^{2}}\right) \sinh \frac{T}{2 \beta}+\frac{1}{T} \cosh \frac{T}{2 \beta}}{\cosh \frac{T}{2 \beta}+\frac{1}{T} \sinh \frac{T}{2 \beta}}$ & $\Omega$ & $\Omega$ \\
\hline
\end{tabular}

$$
T=\sqrt{1-\frac{48\left(1-\varepsilon_{b}\right)}{N_{P e} N_{S c} N_{R e}} \frac{D_{e}}{D_{v}} \frac{1}{1-\gamma-\frac{1}{\rho_{c}}\left(1+\frac{1}{\lambda \rho_{c}}\right)}}, \quad \Omega=\frac{1}{T}+\frac{\eta}{T} \frac{1-\frac{3 \lambda \rho_{c}^{2}}{1+\lambda \rho_{c}}+\frac{3 \lambda^{2} \rho_{c}-\lambda^{2} \rho_{c}^{2}+\lambda \rho_{c}-1}{\left(1+\lambda \rho_{c}\right)^{2}} \rho_{c}^{3}}{\left[1-(1-\gamma) \frac{\lambda \rho_{c}^{2}}{1+\lambda \rho_{c}}\right]^{2}}
$$

$$
\begin{aligned}
& \gamma\left(\frac{\partial c_{i}}{\partial \rho}\right)_{\rho=1}=C-\left(c_{i}\right)_{\rho=1} \\
& C(0, \tau)=\delta(\tau=0) \\
& \left(\frac{\partial C}{\partial x}\right)_{x=1}=0
\end{aligned}
$$

The Laplace transform of the output is obtained as

$$
\bar{C}_{e}=\frac{2 \Psi e^{1 / 2 \beta}}{\left(\frac{1}{2 \beta}+\Psi\right) e^{\Psi}-\left(\frac{1}{2 \beta}-\Psi\right) e^{-\Psi}}
$$

where

$$
\begin{aligned}
\Psi= & \frac{1}{2 \beta}\left[1+4 \beta\left\{s+\frac{3 \eta}{\kappa \gamma}\right.\right. \\
& \left.\left.\times\left(1-\frac{1}{1-\gamma+\gamma \sqrt{\kappa s+\phi^{2}} \operatorname{coth} \sqrt{\kappa s+\phi^{2}}}\right)\right\}\right]^{1 / 2}
\end{aligned}
$$

For Case 2, instead of Eq.(7) the following boundary condition is to be used.

$$
C=0, \text { at } x=\infty
$$

The solution in the Laplace domain is

$$
\bar{C}_{e, i n f}=e^{(1 / 2 \beta)-T}
$$

As shown in Table 1, the moments for Case 1 are never identical with those for Case 2.

When $\beta$ is sufficiently small, however, there are no differences in absolute moments between these two cases.

The solution in the time domain is obtained ${ }^{3)}$ by the change of variables in the system of Eqs. (1) to (7),

$$
W=C e^{-x / 2 \beta}, \quad w=c_{i} e^{-x / 2 \beta}
$$

followed by the Fourier transform

$$
\begin{aligned}
& G_{n}=\int_{0}^{1} W \sin \alpha_{n} x d x \\
& \text { inverse transform, } W=2 \sum_{n=1}^{\infty} \frac{\sin \alpha_{n} x}{1-\frac{\sin 2 \alpha_{n}}{2 \alpha_{n}}} G_{n}
\end{aligned}
$$

with similar expression for $w$, and finally by Laplace transform with regard to $\tau$.

The output is

$$
\begin{aligned}
C_{e}= & 2 \beta \sum_{n=1}^{\infty} \sum_{j=1}^{\infty} \frac{\alpha_{n} \sin \alpha_{n}}{1-\frac{\sin 2 \alpha_{n}}{2 \alpha_{n}}} \\
& \times \frac{(1-\gamma) \sin z_{j}+\gamma z_{j} \cos z_{j}}{A_{n, j} \sin z_{j}+B_{n, j} \cos z_{j}} e^{-\left\{2 \beta\left(z_{j}^{2}+\phi^{2}\right) \tau-\kappa\right\} / 2 \kappa \beta}
\end{aligned}
$$


where

$$
\begin{aligned}
& A_{n, j}=1-\gamma+\frac{3 \eta}{2}+\frac{\kappa \gamma}{2}\left(\frac{1}{4 \beta}+\beta \alpha_{n}^{2}-\frac{z_{j}^{2}+\phi^{2}}{\kappa}\right) \\
& B_{n, j}=\gamma z_{j}-\frac{\kappa}{2 z_{j}}\left(\frac{1}{4 \beta}+\beta \alpha_{n}^{2}-\frac{z_{j}^{2}+\phi^{2}}{\kappa}\right)
\end{aligned}
$$

$\alpha_{n}$ is a root of

$$
\tan \alpha_{n}=-2 \beta \alpha_{n}
$$

and $z_{j}$ is a root of

$$
\tan z_{j}=-z_{j} \frac{\gamma\left(\frac{1}{4 \beta}+\beta \alpha_{n}^{2}-\frac{z_{j}^{2}+\phi^{2}}{\kappa}\right)+\frac{3 \eta}{\kappa}}{(1-\gamma)\left(\frac{1}{4 \beta}+\beta \alpha_{n}^{2}-\frac{z_{j}^{2}+\phi^{2}}{\kappa}\right)-\frac{3 \eta}{\kappa}}
$$

\section{(B) Shell Reaction Model}

This model has been applied to many non-catalyzed solid-gas reactions. Let us consider a bed packed with particles consisting of non-porous unreacted core of radius $\rho_{c}$ (in non-dimension) and porous ash layer outside the core. Because the amount of injected gas reactant is small, the core radius will be kept unchanged. If the reaction at the core surface is of isothermal first order with regard to the gas reactant, the system is expressed as Eq.(1) and

$$
\kappa \frac{\partial c_{i}}{\partial \tau}=\frac{\partial^{2} c_{i}}{\partial \rho^{2}}+\frac{2}{\rho} \frac{\partial c_{i}}{\partial \rho}, \text { for } \rho_{c}<\rho<1
$$

with Eqs.(3) to (7) and

$$
\left(\frac{\partial c_{i}}{\partial \rho}\right)_{\rho_{c}}=\lambda\left(c_{i}\right)_{\rho_{c}}
$$

The Laplace transform of the output is

$$
\overline{C_{e}}=2 \Gamma e^{1 / 2 \beta} /\left\{\left(\frac{1}{2 \beta}+\Gamma\right) e^{\Gamma}-\left(\frac{1}{2 \beta}-\Gamma\right) e^{-\Gamma}\right\}
$$

where

$$
\begin{aligned}
\Gamma & =\frac{1}{2 \beta} \sqrt{1+4 \beta\left[s+\frac{3 \eta}{\kappa \gamma}(1-b)\right]} \\
b & =\frac{a+\tanh \sqrt{\kappa s}}{\gamma \sqrt{\kappa s}+a(1-\gamma)+(1-\gamma+a \gamma \sqrt{\kappa s}) \tanh \sqrt{\kappa s}} \\
a & =\frac{\rho_{c} \sqrt{\kappa s}-\left(1+\lambda \rho_{c}\right) \tanh \rho_{c} \sqrt{\kappa s}}{1+\lambda \rho_{c}-\rho_{c} \sqrt{\kappa s} \tanh \rho_{c} \sqrt{\kappa s}}
\end{aligned}
$$

For Case 2 the solution in the Laplace domain is

$$
\bar{C}_{e, i n f}=e^{(1 / 2 \beta)-\Gamma}
$$

Similarly to (A), as compared in Table 2, there are differences in moments between Case 1 and Case 2.

The solution in the time domain is obtained by the same procedures as before.

$$
\begin{aligned}
C_{e}= & 2 \beta \sum_{n=1}^{\infty} \sum_{j=1}^{\infty} \\
& \frac{\alpha_{n} \sin \alpha_{n}}{1-\frac{\sin 2 \alpha_{n}}{2 \alpha_{n}}} \frac{P_{j} \sin z_{j}+Q_{j} \cos z_{j}}{U_{n, j} \sin z_{j}+V_{n, j} \cos z_{j}} e^{-\left(2 \beta z_{j} z_{-}-\kappa\right) / 2 \kappa \beta}
\end{aligned}
$$

where $P_{j}=1-\gamma-\gamma F_{j} z_{j}$

$$
\begin{aligned}
Q_{j}= & \gamma z_{j}+F_{j}(1-\gamma) \\
U_{n, j}= & P_{j}+a_{n, j}\left(\frac{\kappa F_{j}}{2 z_{j}}+\frac{\kappa \gamma}{2}-\gamma q_{j} z_{j}\right) \\
& +3 \eta\left(\frac{1}{2}-\frac{q_{j} z_{j}}{\kappa}\right) \\
V_{n, j}= & Q_{j}+a_{n, j}\left[q_{j}(1-\gamma)+\frac{\kappa \gamma F_{j}}{2}-\frac{\kappa}{2 z_{j}}\right] \\
& -3 \eta\left(\frac{q_{j}}{\kappa}-\frac{F_{j}}{2}\right) \\
a_{n, j}= & \frac{1}{4 \beta}+\beta \alpha_{n}^{2}-\frac{z_{j}^{2}}{\kappa} \\
F_{j}= & \frac{\rho_{c} z_{j}-\left(1+\rho_{c} \lambda\right) \tan \rho_{c} z_{j}}{1+\rho_{c} \lambda+\rho_{c} z_{j} \tan \rho_{c} z_{j}} \\
q_{j}= & \frac{\kappa \rho_{c}}{2 z_{j}}\left[1-\frac{1-F_{j}\left(\rho_{c} z_{j}-\rho_{c} \lambda \tan \rho_{c} z_{j}\right)}{1+\rho_{c} \lambda+\rho_{c} z_{j} \tan \rho_{c} z_{j}}\right]
\end{aligned}
$$

$\alpha_{n}$ is a root of Eq.(16), and $z_{j}$ is a root of

$$
\tan z_{j}=-\frac{a_{n, j} Q_{j}+\frac{3 \eta}{\kappa}\left(z_{j}-F_{j}\right)}{a_{n, j} P_{j}-\frac{3 \eta}{\kappa}\left(1+F_{j} z_{j}\right)}
$$

\section{Nomenclature}

$=$ dimensionless tracer concentration in bulk stream; (concentration)/(amount of tracer input/ extraparticle void volume of bed)

$\begin{array}{ll}C_{e} & =C \text { at reactor exit } \\ \bar{C}_{e} & =\text { Laplace transform of } C_{e}\end{array}$

$=$ Laplace transform of $C_{e}$ [-]

$c_{i}=$ dimensionless tracer concentration in particle; (concentration)/(amount of tracer input/ extraparticle. void volume of bed)

$=$ axial fluid mixing coefficient based on extraparticle void area

$=$ effective diffusivity in porous solid

$D_{p} \quad=$ particle diameter $\quad[\mathrm{cm}]$

$D_{v} \quad=$ molecular diffusivity $\quad\left[\mathrm{cm}^{2} / \mathrm{sec}\right]$

$\left[\mathrm{cm}^{2} / \mathrm{sec}\right]$
$\left[\mathrm{cm}^{2} / \mathrm{sec}\right]$

$k_{f} \quad=$ particle-fluid mass transfer coefficient $\quad[\mathrm{cm} / \mathrm{sec}]$

$k_{s} \quad=$ reaction rate constant for shell reaction model

$[\mathrm{cm} / \mathrm{sec}]$

$k_{v} \quad=$ reaction rate constant for homogeneous reaction model

$=$ packed bed height

$\begin{array}{ll}L & =\text { packed bed } \\ m_{n} & =n \text {-th moment }\end{array}$

$N_{P e} \quad=D_{p} U / D_{a x}$

$N_{R e} \quad=D_{p \varepsilon_{b}} U / \nu$

$N_{S c} \quad=\nu / D_{v}$

$N_{S h} \quad=k_{f} D_{p} / D_{v}$,

$R \quad=$ particle radius

$=$ radial distance variable

$=$ radius of unreacted core

$=$ time

$=$ interstitial fluid velocity

$=$ axial distance variable

$=X / L$

$=$ root of Eq.(17) or (33)

$[1 / \mathrm{sec}]$

[cm]

$[-]$

$[-]$

$[-]$

$[-]$

$[-]$

$[\mathrm{cm}]$

$[\mathrm{cm}]$

[cm]

[sec]

$[\mathrm{cm} / \mathrm{sec}]$

[cm]

$[-]$

$=$ root of Eq.(16)

[-]

$=1 / N_{P e}\left(\frac{L}{D_{p}}\right)$

$[-]$

$=2\left(\frac{D_{e}}{D_{v}}\right) / N_{S h}$

$=$ Kronecker's delta function

= extraparticle void fraction

$=$ void fraction in particle for homogeneous reaction 


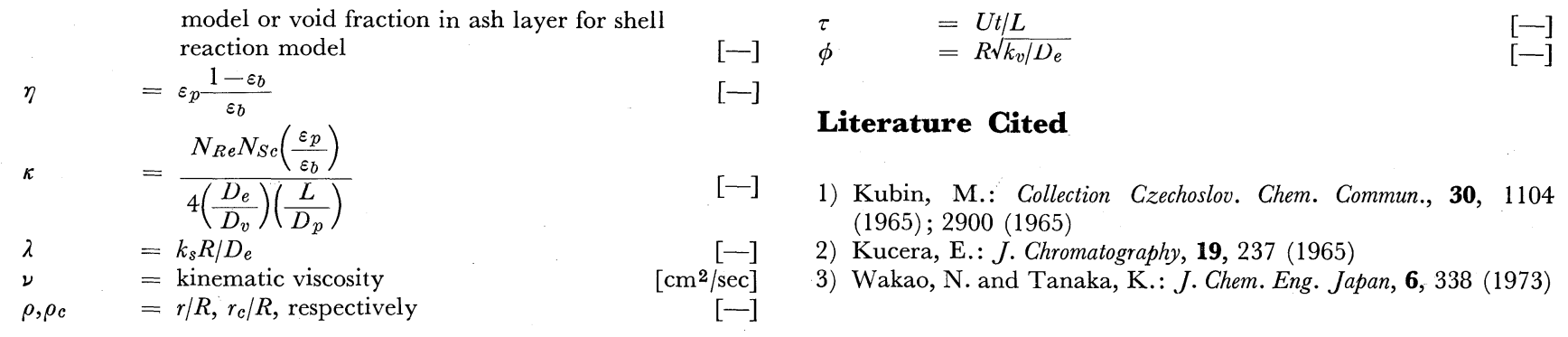

